\section{Vol. 64, No. 52}

In the report, "Diagnosis of Tuberculosis in Three Zoo Elephants and a Human Contact — Oregon, 2013," on page 1398, the first sentence of the second paragraph should have read as follows: "In May 2013, a routine annual culture of a sample from a trunk washing on elephant A, an Asian elephant aged 30 years at a zoo in Oregon's Multnomah County, yielded Mycobacterium tuberculosis, indicating active, potentially infectious disease."

\section{Vol. 64, No. 25}

In the report, "Monitoring of Persons with Risk for Exposure to Ebola Virus Disease - United States, November 3, 2014-March 8,2015," on page 688, an error occurred in "FIGURE 3. Number of persons with potential Ebola exposure monitored in 50 states, New York City, and the District of Columbia - November 3, 2014-March 8, 2015." The shading for the District of Columbia (DC) should have indicated that 200-499 persons were monitored in DC. 